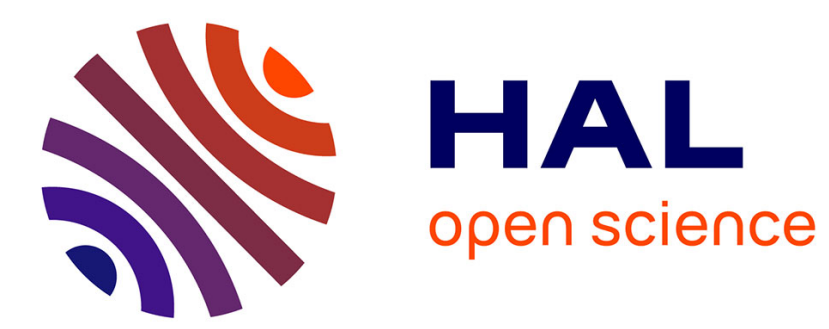

\title{
Preferential hydration fully controls the renaturation dynamics of collagen in water-glycerol solvents
}

\author{
Olivier Ronsin, C. Caroli, Tristan Baumberger
}

\section{To cite this version:}

Olivier Ronsin, C. Caroli, Tristan Baumberger. Preferential hydration fully controls the renaturation dynamics of collagen in water-glycerol solvents. European Physical Journal E: Soft matter and biological physics, 2017, 40 (5), pp.55. 10.1140/epje/i2017-11545-1 . hal-01480576

\section{HAL Id: hal-01480576 https://hal.science/hal-01480576}

Submitted on 1 Mar 2017

HAL is a multi-disciplinary open access archive for the deposit and dissemination of scientific research documents, whether they are published or not. The documents may come from teaching and research institutions in France or abroad, or from public or private research centers.
L'archive ouverte pluridisciplinaire HAL, est destinée au dépôt et à la diffusion de documents scientifiques de niveau recherche, publiés ou non, émanant des établissements d'enseignement et de recherche français ou étrangers, des laboratoires publics ou privés. 


\title{
Preferential hydration fully controls the renaturation dynamics of collagen in water-glycerol solvents
}

\author{
O. Ronsin, C. Caroli, and T. Baumberger \\ Institut des Nanosciences de Paris, Sorbonne universités, \\ UPMC univ Paris 6 and CNRS-UMR 7588, 4 place Jussieu, F-75252 Paris, France
}

\begin{abstract}
Glycerol is one of the additives which stabilize collagen, as well as globular proteins, against thermally induced denaturation - an effect explained by preferential hydration, i.e. by the formation, in water/glycerol solvents, of a hydration layer whose entropic cost favors the more compact triplehelix native structure against the denatured one, gelatin. Quenching gelatin solutions promotes renaturation which, however, remains incomplete, as the formation of a gel network gives rise to growing topological constraints. So, gelatin gels exhibit glass-like dynamical features such as slow aging of their shear modulus and stretched exponential stress relaxation, the study of which gives us access to the re(de)naturation dynamics of collagen. We show that this dynamics is independent of the bulk solvent viscosity and controlled by a single parameter, the undercooling $\Delta T$ below the glycerol-concentration dependent denaturation temperature. This provides direct proof of (i) the presence of a nanometer thick, glycerol-free hydration layer (ii) the high locality of the kinetically limiting process governing renaturation.
\end{abstract}

\section{INTRODUCTION}

Glycerol has been for decades one of the most widely used additives that stabilize the native structure of proteins against denaturation induced by e.g. thermal stresses. Extensive work by Timasheff and collaborators [1] on globular proteins led them to explain this effect in terms of the so-called preferential hydration effect: in a stabilizing binary solvent, the protein interacts more favorably with water than with the cosolvent. This results in the formation of a hydration shell out of which the cosolvent is excluded, at the expense of a mixing entropy cost. The resultant increase in chemical potential of the protein acts as a driving force to minimize the protein-solvent interfacial area, leading to stabilization of the (more compact) native conformation. This interpretation was later shown [2-4] to be valid as well for collagen. Collagen molecules are constituted of the wrapping into a right-handed helix of three left-handed single strand $\alpha$-like (polyproline II) helices. When heating a collagen solution above $T_{\text {denat }}$, denaturation of this $\mathrm{H}$-bond stabilized structure results in a so-called gelatin solution of single-stranded chains in the coil configuration - hence an increase of the polymer/solvent area of contact. It has been found $[2,4]$ that the increase $\Delta T_{\text {denat }}$ upon addition of glycerol grows linearly with its concentration $\phi$, up to $\phi \simeq 40 \mathrm{wt} \%$.

Renaturation of collagen from a gelatin solution occurs via nucleation and growth of triple-helix segments $[5,6]$, a process kinetically controlled by the unusually slow cistrans isomerisation of the prolyl peptide bonds present in large proportion along the chains [7]. However, except for very small gelatin concentrations $(\lesssim 1 \%)$ renaturation is only partial. Indeed, each chain is engaged in the formation of several triple-helix nuclei, each of which connects it with different partners. The nucleation and growth of these cross-links leads to the formation of a percolating thermoreversible gelatin gel network.
This process is revealed by the growth with time $t$ of the shear elastic modulus $G$. After a lapse time $t_{0}$ associated with percolation of the polymer network, $G$ first exhibits a rapid increase due to further nucleation and growth of cross-links [8, 9]. It then crosses over to a slow regime in which $G$ grows logarithmically with time, without any measurable trend towards saturation [10]. In this glass-like aging stage, increasingly strong topological constraints prevent the formation of new cross-links, and logarithmic strengthening can be assigned to network rearrangements taking place via thermally activated $\mathrm{H}$ bonds fluctuations resulting in zippings and unzippings of the existent triple-helix segments $[5,11]$.

The same type of rearrangements also control the stress relaxation dynamics of gelatin gels [12]. In such experiments it is found that, upon applying a shear deformation step, which biases the glass-like complex energy landscape of the network, the macroscopic shear stress exhibits a slow, stretched exponential decrease $\grave{a}$ la Kohlrausch [13] with a characteristic time growing as a power of the gel age $t_{w}$, i.e. the time elapsed since the beginning of the thermal quench.

In short, monitoring the aging of the elastic modulus and/or the stress relaxation of gelatin gels yields information about the dynamical scale of the processes which control the re(de)naturation of collagen.

In this article we study gelatin gels formed in waterglycerol solvents and assess the evolution of their slow dynamics with glycerol concentration. We show that this dynamics is by no means slaved by the viscosity of the bulk solvent but fully controlled by the presence of the water shell associated with Timasheff's preferential hydration effect. Conversely, this result confirms the locality of the zipping-unzipping processes responsible for gelatin aging and stress relaxation. 


\section{MATERIALS AND METHODS}

Samples are prepared by dissolving, at $55^{\circ} \mathrm{C}, 5$ wt $\%$ gelatin (300 Bloom, from porcine skin, Sigma) in either pure water or mixtures of water and glycerol at concentration $\phi=40$ or 60 wt $\%$, of viscosity $\eta(\phi)$ (see Fig. 4). Gelatin solutions in pure water have a $\mathrm{pH}=5.1 \pm 0.05$ at $40^{\circ} \mathrm{C}$.

The pregel solution is poured into the sand-blasted cone-plate cell of a stress-controlled rheometer (AntonPaar, MCR 501) protected against solvent evaporation by a paraffin oil rim. The sample temperature is first set at $55^{\circ} \mathrm{C}$ and then ramped at $7.5^{\circ} \mathrm{C} / \mathrm{min}$ down to the working temperature $T$ controlled to within $\pm 0.1^{\circ} \mathrm{C}$.

\section{A. Determination of the gelation temperature $T_{g e l}$}

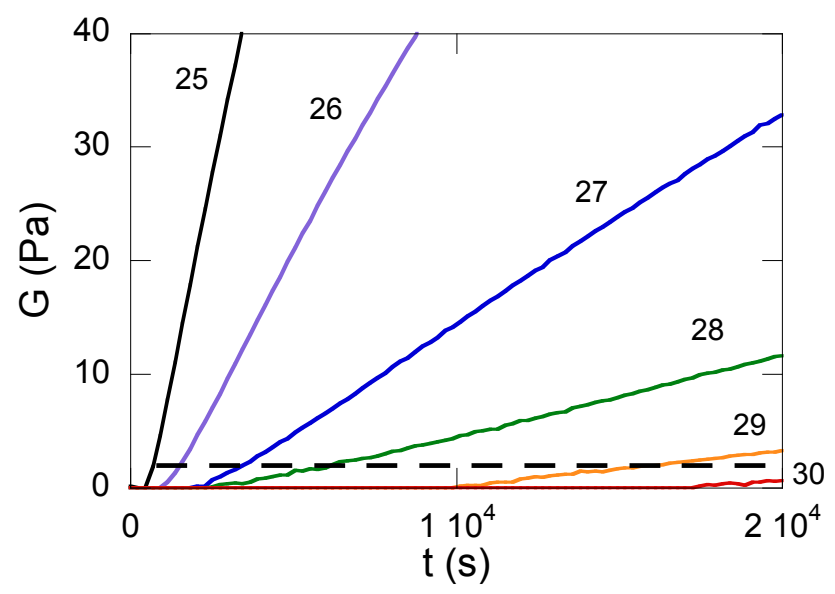

FIG. 1. Early time evolution of the shear modulus for a $\phi=0$ (pure water) gel at temperatures $\left(\right.$ in ${ }^{\circ} \mathrm{C}$ ) as specified by the labels. The dashed line indicates the $G=2 \mathrm{~Pa}$ threshold chosen to define the gelation temperature. Here, $T_{\text {gel }}=30^{\circ}$ C.

The early advancement of the gelation process is monitored by measuring the growth of the storage shear modulus $G$ at frequency $1 \mathrm{~Hz}$ versus the time $t$ elapsed after reaching the working temperature $T$. For this particular set of experiments we use a strain amplitude of $4 \%$, a relatively large value which we choose in order to optimize the signal-to-noise ratio while remaining in the linear mechanical response domain.

Successive runs are performed at increasing temperatures spaced by $1^{\circ} \mathrm{C}$. After each run, the sample is reinitialized by reheating it up to $55^{\circ} \mathrm{C}$ and shearing at the constant rate $1 \mathrm{~s}^{-1}$ for $200 s$ before performing a new quench. We have checked that this protocol ensures that mechanical measurements are reproducible to within $1 \%$, over 20 cycles at least.

At low enough temperatures, after an initial induction period $t_{0}$ during which it remains below the detection threshold, a gel network percolates and $G$ starts growing. As seen on Fig.1, $t_{0}$ increases with temperature. We define $T_{g e l}$ as the lowest temperature at which $G\left(t=2 \times 10^{4}\right.$ s) does not exceed $2 \mathrm{~Pa}$, a threshold value much smaller than the typical moduli (several hundred $\mathrm{Pa}$ ) used in this study. With this protocol $T_{g e l}$ is thus determined to within $1^{\circ} \mathrm{C}$.

\section{B. Mechanical measurements}

For a given value of $\phi$, time evolution of the modulus and stress relaxation experiments are performed on a single sample, reinitialized between various runs as described above.

The time-dependent storage modulus $G(t)$ is measured at frequency $1 \mathrm{~Hz}$ with a strain amplitude $1 \%$.

Stress relaxation experiments are performed as follows. We first let gelation proceed freely for a time $t_{w}$ (defined as the age of the gel) after the end of the quench, then apply a $5 \%$ strain step and monitor the subsequent evolution of the shear stress $\sigma(t)$. Such measurements have been performed for $t_{w}=200,10^{3}$ and $10^{4} \mathrm{~s}$.

\section{RESULTS AND DISCUSSION}

In order to get some insight into the effect of glycerol on the dynamics of collagen re(de)naturation, we first performed the following preliminary experiments, in which we compare the transient mechanical behavior of gels with $\phi=0$ (pure water) and $\phi=60 \%$ solvents, at room temperature $T=20^{\circ} \mathrm{C}$ where gelatin gelation is known to proceed rapidly.

A simple macroscopic argument leads to expect the characteristic time scale(s) of relaxation processes to scale as the viscosity $\eta_{\phi}$ of the solvent[14]. Such a guess is in particular suggested by two groups of previous results which have both shown this scaling to hold for :

(i) the linear viscoelastic loss angle measured in a frequency range chosen so as to probe the mobility of individual strands of the gel network[15].

(ii) the rate dependence of the gel fracture energy. For gelatin gels, this process occurs without any scission of the polymer chains, but via their stress-induced disentanglement and pull-out[16, 17].

Fig.2 displays the stress relaxation curves of the two gels, the compositions of which was chosen so that they exhibit the largest viscosity contrast $\left(\eta_{60} / \eta_{0}=11\right.$, see inset of Fig. 4). Since it is known that the characteristic time of the stretched exponential stress relaxation of pure water gels scales as a power of the gel age $t_{w}$, both measurements have been performed at the same $t_{w}=10^{4} \mathrm{~s}$. In order to assess the above scaling expectation, we also plotted on the same figure the normalized stress $\sigma / \sigma_{0}$ (with $\sigma_{0}$ the stress immediately after loading) vs. the scaled time $\left(\eta_{0} / \eta_{60}\right) t$. Obviously, our naive guess is not correct. On the contrary, visual inspection suggests that 
the stress relaxation dynamics is but very weakly slowed down by the presence of glycerol.

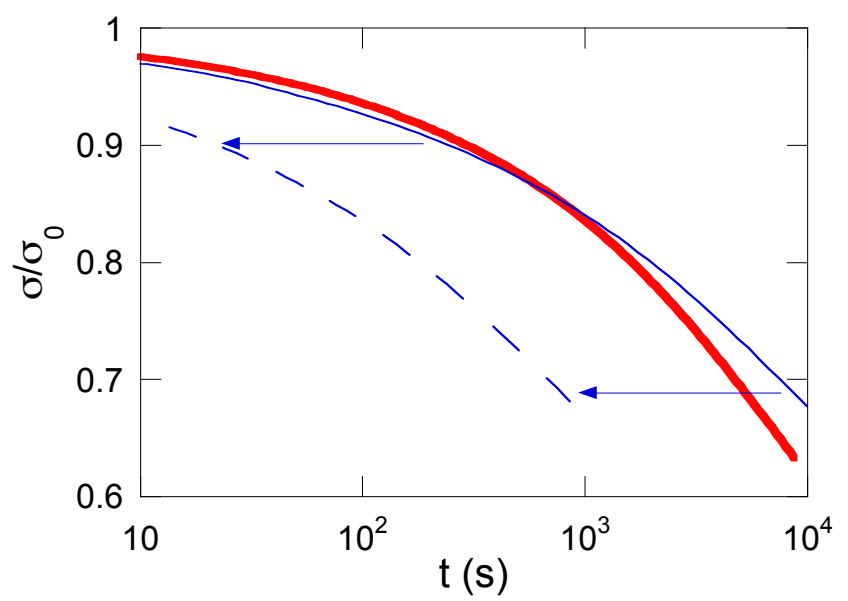

FIG. 2. Stress relaxation curves at $20^{\circ} \mathrm{C}$ for gels with age $t_{w}=10^{4} \mathrm{~s}$ and glycerol content $\phi=0$ (red, thick line) and $\phi=60 \mathrm{wt} \%$ (blue, thin line). The blue, dashed line shows the $\phi=60 \mathrm{wt} \%$ data plotted vs. scaled time $\left(\eta_{0} / \eta_{60}\right) t$. Arrows indicate the corresponding shift.

A second piece of information is provided by comparing the growth dynamics of the moduli of the two gels (Fig.3). Strikingly, $G$ is seen to grow noticeably faster for the more viscous gel - an effect which is dramatically enhanced when scaling the time variable.

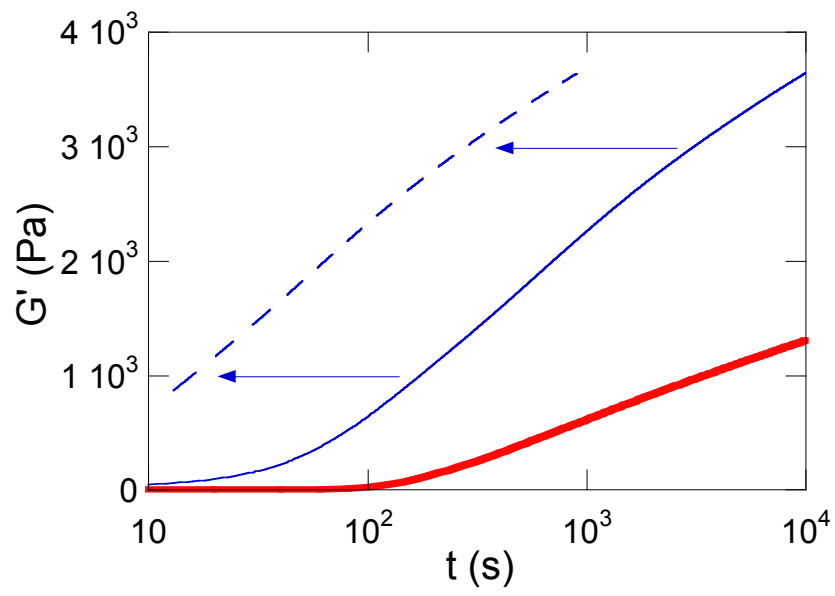

FIG. 3. Shear modulus vs. time after quench for gels at $20^{\circ} \mathrm{C}$ with $\phi=0$ (red, thick line) and $\phi=60 \mathrm{wt} \%$ (blue, thin line). The blue, dashed line shows the $\phi=60 \mathrm{wt} \%$ data plotted vs. scaled time $\left(\eta_{0} / \eta_{60}\right) t$. Arrows indicate the corresponding shift.

Clearly, a hydrodynamic description of the dissipation associated with the movements of the network responsible for aging and stress relaxation in terms of the viscosity of the bulk solvent is inappropriate to account for our observations. We must therefore consider the possibility for the microscopic structuration of the solvent to be of crucial importance. This is strongly suggested by the nature of the gelation process: it involves the formation of triple helix cross-links exhibiting the structure of native collagen which, as mentioned above, induces preferential hydration as revealed by the dependence of the denaturation temperature $T_{\text {denat }}$ on the glycerol content.

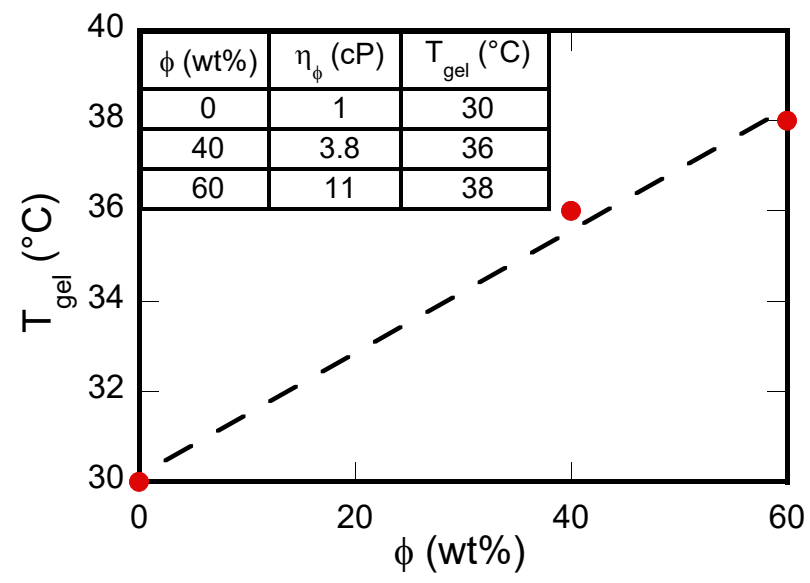

FIG. 4. Gelation temperature of $5 \mathrm{wt} \%$ gelatin gels in aqueous solvents with glycerol weight fraction $\phi$. The insert shows the solvent viscosity $\eta_{\phi}$ at $T=20^{\circ} \mathrm{C}$ and $T_{g e l}(\phi)$ values.

Since gelation means renaturation, $T_{\text {gel }}$ should be a good indicator of $T_{\text {denat }}$. In order to test this for our gels, we have measured $T_{\text {gel }}(\phi)$ (see Materials and Methods). Fig.4 shows that $T_{\text {gel }}$ grows linearly with the glycerol content with a slope

$$
\frac{d T_{g e l}}{d \phi}=0.14^{\circ} \mathrm{C} / \mathrm{wt} \%
$$

Both the large extent of the linear behavior (which we extend here up to $60 \mathrm{wt} \%$ ) and the slope value are fully compatible with the results of Gekko and Koga [2] and Penkova et al [4] for the $\phi$-dependence of the denaturation temperature $T_{\text {denat }}\left(d T_{\text {denat }} / d \phi=0.12 \pm\right.$ $0.01^{\circ} \mathrm{C} / \mathrm{wt} \%$ for calf skin collagen). From now on we will therefore use $T_{\text {gel }}$ as an approximant for $T_{\text {denat }}$.

We now want to identify the parameters which controls the modulus build up and stress relaxation dynamics at various temperatures and glycerol concentrations. These mechanical responses are governed by the dynamics of the microscopic motions of network constituents involved in the de(re)naturation transition. We note that in the temperature range $\left(\left(T_{\text {gel }}-T\right) / T \ll 1\right)$ relevant to our experiments, the thermodynamic force which drives these motions is controlled, not by temperature itself, but by the undercooling $\Delta T=T_{g e l}(\phi)-T$.

In order to determine to which extent $\Delta T$ is effectively a relevant control parameter for the dynamics, we have compared the early build up of the shear modulus of a 
$\phi=40 \mathrm{wt} \%$ gel at $30^{\circ} \mathrm{C}$ (i.e with $\Delta T=6^{\circ} \mathrm{C}$ ) and of a $\phi=0$ one at temperatures $T$ ranging from 22 to $26^{\circ} \mathrm{C}$ ( $\Delta T$ from 8 to $4^{\circ} \mathrm{C}$ ). As can be seen on Fig.5, the five $\phi=0$ curves are widely splayed and the one among them which lies closest to the $\phi=40$ one corresponds to the same $\Delta T=6^{\circ} \mathrm{C}$ value.

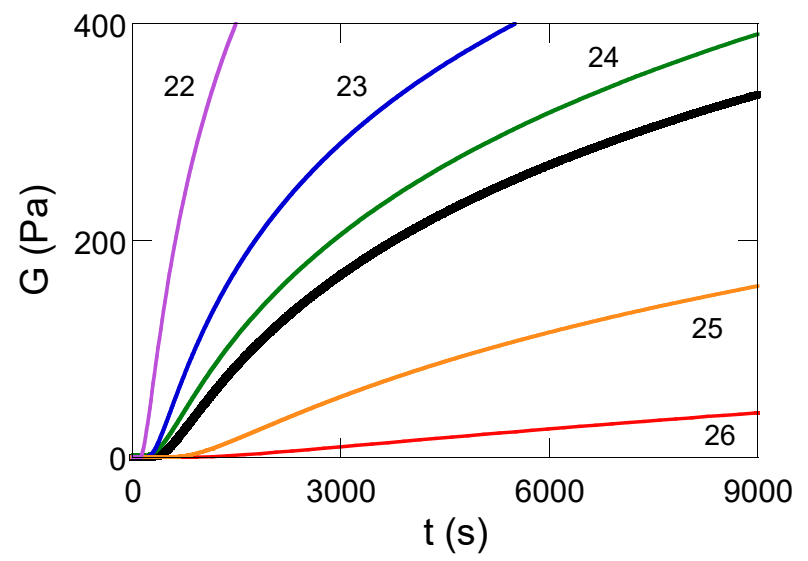

FIG. 5. Early growth of the shear modulus $G$ of a $\phi=40$ gel at $T=30^{\circ} \mathrm{C}\left(\Delta T=6^{\circ} \mathrm{C}\right)$ : black, thick line, as compared with a $\phi=0$ gel at various temperatures in ${ }^{\circ} \mathrm{C}$, as labelled: color, thin lines.

With this observation in mind, we have performed, for each of the three $\phi$ values, measurements of modulus build up and stress relaxation over extended time ranges, for three fixed values of $\Delta T\left(6,10,15^{\circ} \mathrm{C}\right)$.

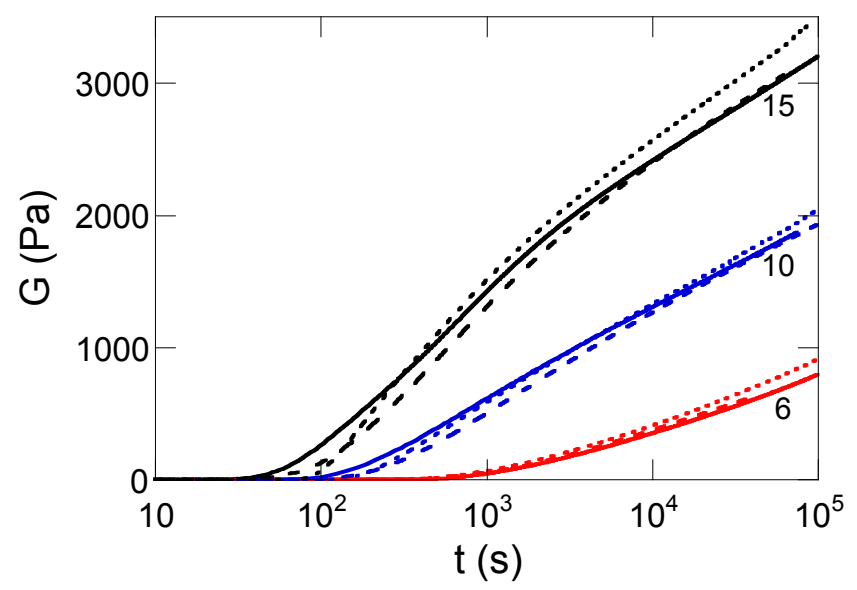

FIG. 6. Modulus build up for solvents with glycerol contents $\phi=0$ (full lines), 40 (dotted lines) and $60 \mathrm{wt} \%$ (dashed lines). Each group of curves corresponds (see labels) to a common distance below the gelation temperature: from top to bottom (see labels) $\Delta T=15$ (black), 10 (blue) and $6^{\circ} \mathrm{C}$ (red).

Results for the evolution of $G$ are shown on Fig.6. Strikingly, for each value of $\Delta T$, the curves for the three solvent compositions collapse very nicely over the full time range. That is, $\Delta T$ controls the modulus build up dynamics not only in the early stage of gelation corresponding to nucleation and initial cross-link growth but, as well, in the late logarithmic aging regime resulting from cross-link rearrangements via zipping-unzipping of the collagen segments.
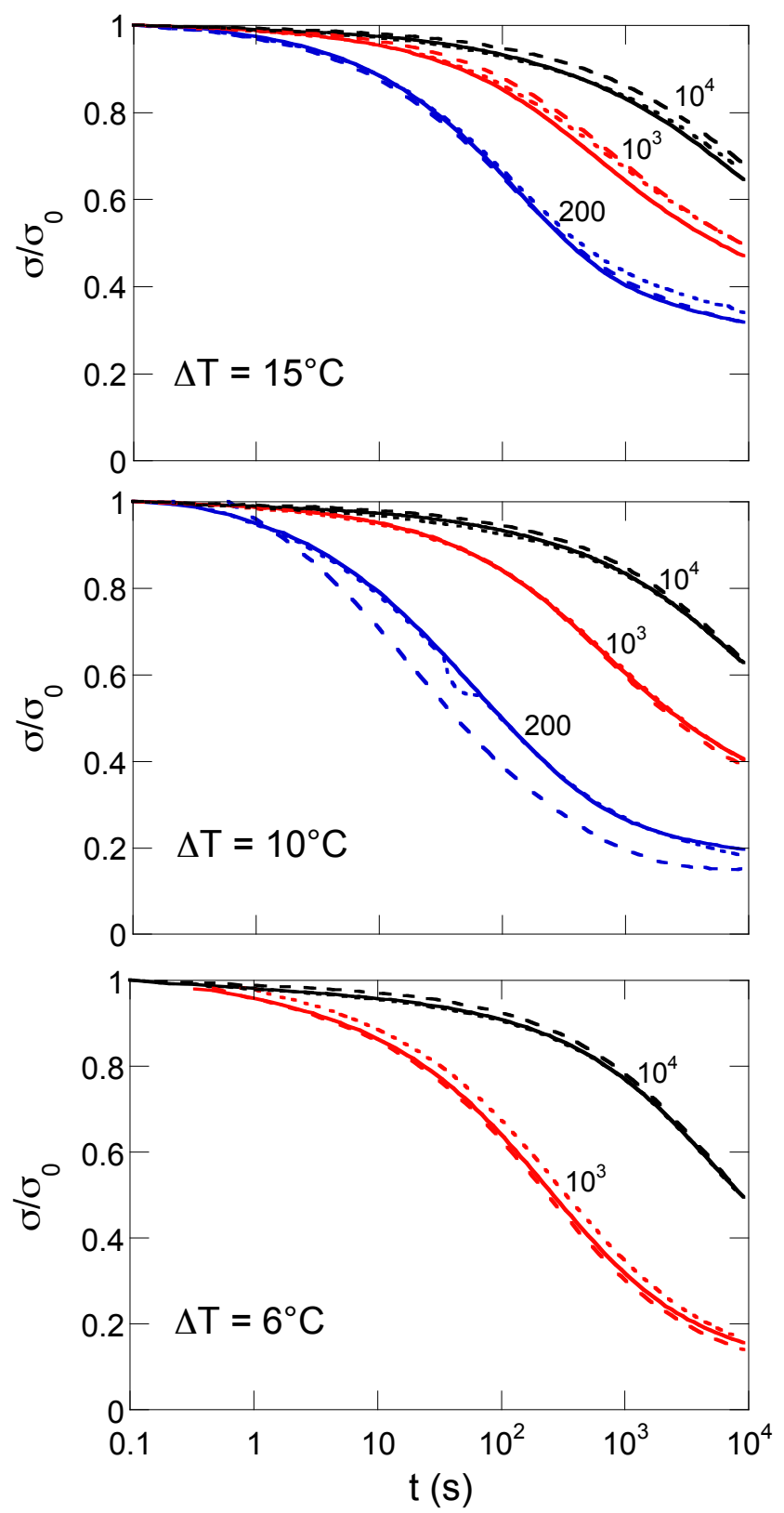

FIG. 7. Stress relaxation curves for solvents with glycerol contents $\phi=0$ (full lines), 40 (dotted lines) and $60 \mathrm{wt} \%$ (dashed lines). Each panel corresponds to a fixed value of $\Delta T$, as indicated. Each group of curves corresponds (see labels) to a common age value: $t_{w}=200 \mathrm{~s}$ (blue), $10^{3} \mathrm{~s}$ (red) and $10^{4}$ $\mathrm{s}$ (black). Note the absence of $t_{w}=200 \mathrm{~s}$ data in the lower panel, due to the fact that at this value of $\Delta T\left(6^{\circ} \mathrm{C}\right)$, network percolation has not yet occurred. 
Each of the three panels of Fig.7 displays stress relaxation curves measured, for a given $\Delta T$, at different stages of gel aging $\left(t_{w}=200,10^{3}\right.$ and $\left.10^{4} \mathrm{~s}\right)$. Again, for each value of $\Delta T$ and $t_{w}$, the data for the three solvent compositions exhibit excellent collapse over 5 time decades.

These two groups of results clearly demonstrate that, in a substantial temperature range of biological relevance, the de(re)naturation dynamics of collagen is controlled by a single parameter, namely the undercooling $\Delta T$ below the denaturation temperature. This dynamics is affected by the presence of glycerol exclusively via the $\phi$ dependent upward shift of $T_{\text {denat }}$, and is completely insensitive to the value of the bulk solvent viscosity.

\section{CONCLUSIONS}

This behavior entails the following conclusions.

(i) The time-dependent network structure of gelatin gels formed in water/glycerol mixtures is uniquely determined, at all stages of its evolution, by the undercooling $\Delta T$ below the glycerol-concentration dependent gelation temperature.

(ii) Renaturation in a water-glycerol mixture proceeds as it would in pure water. This dynamical behavior directly confirms the presence of a hydration layer and indicates that glycerol is fully expelled out of it.

(iii) At a given value of $\Delta T$, i.e. for a fixed chemical potential difference between the native and denatured states, the probability of the zipping-unzipping elementary events, i.e. of the (triple helix) $\leftrightarrow$ (three coils) thermally activated transition, is glycerol-insensitive. Hence the free energy barrier height is $\phi$-independent. The same is true of the frequency of the vibration modes which control the prefactor of the Eyring activation exponential. In view of the fact that the kinetically limiting process is known to be the cis-trans isomerisation of Gly-Pro bonds[7], we are led to suggest that the thickness of the glycerol-free hydration layer should range close to one nanometer.

Finally, putting these conclusions in regard with the above-mentioned results about the viscoelastic loss angle and the rate dependence of the fracture energy leads us to formulate a last, more general, remark.

Note that, on the one hand, the dynamics which we have studied here is controlled by the ultra local, subnanometric, motions which trigger network rearrangements. On the other hand, in ref [15] the authors chose to probe preferentially the frictional dynamics of the flexible inter-crosslink strands, which involve motions on the scale of the mesh size $\xi$ of the gel network. Similarly, $\xi$ is the spatial scale relevant to dissipation associated with crack propagation. Indeed, the velocity dependence of the fracture energy is due to the viscous dissipation induced by the pull out of gelatin chains out of the poroelastic network $[16,17]$. In this process, the solvent is sheared on a space range on the order of the gel mesh size. For commonly used gels, $\xi$ lies in the $10 \mathrm{~nm}$ range - i.e. extends much beyond the hydration range. This explains why the dissipation measured in the the two latter experiments is controlled by the bulk solvent viscosity rather than by that of the hydration layer.

In summary, from the results of our study of the effect of glycerol on the macroscopic dynamic elastic responses of gelatin gels, we conclude, in agreement with Frauenfelder's views about protein motions [18], to the primary importance of the space scale of the motions on protein dynamics.

\section{ACKNOWLEDGMENTS}

We are grateful to M. Djabourov for an enlightening discussion.
[1] S. N. Timasheff, in Linkage Thermodynamics of Macromolecular Interactions, Advances in Protein Chemistry, Vol. 51, edited by E. D. Cera (Academic Press, 1998) pp. $355-432$.

[2] K. Gekko and S. Koga, The Journal of Biochemistry 94, 199 (1983).

[3] G. C. Na, Biochemistry 25, 967 (1986).

[4] R. Penkova, I. Goshev, S. Gorinstein, and P. Nedkov, Food chemistry 66, 483 (1999).

[5] K. te Nijenhuis, "Gelatin," in Thermoreversible Networks: Viscoelastic Properties and Structure of Gels, Advances in Polymer Science (Springer-Verlag Berlin Heidelberg, Berlin, Heidelberg, 1997) pp. 160-193.

[6] M. Djabourov, K. Nishinari, and S. B. Ross-Murphy, Physical gels from biological and synthetic polymers (Cambridge University Press, 2013).

[7] H. P. Bächinger, P. Bruckner, R. Timpl, D. J. Prockop, and J. Engel, European Journal of Biochemistry 106, 619 (1980).
[8] L. Guo, R. H. Colby, C. P. Lusignan, and T. H. Whitesides, Macromolecules 36, 9999 (2003).

[9] L. Guo, R. H. Colby, C. P. Lusignan, and A. M. Howe, Macromolecules 36, 10009 (2003).

[10] V. Normand, S. Muller, J.-C. Ravey, and A. Parker, Macromolecules 33, 1063 (2000).

[11] H. Souguir, O. Ronsin, C. Caroli, and T. Baumberger, Physical Review E 91, 042305 (2015).

[12] M. Miller, J. D. Ferry, F. W. Schremp, and J. E. Eldridge, The Journal of Physical Chemistry 55, 1387 (1951).

[13] O. Ronsin, C. Caroli, and T. Baumberger, Physical review letters 103, 138302 (2009).

[14] E. Evans and K. Ritchie, Biophysical journal 72, 1541 (1997).

[15] J.-L. Laurent, P. A. Janmey, and J. D. Ferry, Journal of Rheology 24, 87 (1980).

[16] T. Baumberger, C. Caroli, and D. Martina, Nature materials 5, 552 (2006). 
[17] T. Baumberger, C. Caroli, and D. Martina, The European Physical Journal E 21, 81 (2006).

[18] H. Frauenfelder, G. Chen, J. Berendzen, P. W. Fenimore, H. Jansson, B. H. McMahon, I. R. Stroe, J. Swenson, and R. D. Young, Proceedings of the National Academy of Sciences 106, 5129 (2009). 